\title{
KDM4B facilitates colorectal cancer growth and glucose metabolism by stimulating TRAF6-mediated AKT activation
}

\author{
Haijie Li ${ }^{\dagger}$, Jingqin Lan ${ }^{\dagger}$, Guihua Wang, Kaixuan Guo, Caishun Han, Xiaolan Li, Junbo Hu, Zhixin Cao and Xuelai Luo*
}

\begin{abstract}
Background: Histone lysine demethylase 4B (KDM4B) has been implicated in various pathological processes and human diseases. Glucose metabolism is the main pattern of energy supply in cells and its dysfunction is closely related to tumorigenesis. Recent study shows that KDM4B protects against obesity and metabolic dysfunction. We realized the significant role of $\mathrm{KDM} 4 \mathrm{~B}$ in metabolism. However, the role of KDM4B in glucose metabolism remains unclear. Here, we sought to delineate the role and mechanism of KDM4B in glucose metabolism in colorectal cancer (CRC).

Methods: We first analyzed the role of KDM4B in glucose uptake and CRC growth. We then investigated the consequences of KDM4B inhibition on the expression of GLUT1 and AKT signaling, also explored the underlying mechanism. Finally, we detected the mechanism in vivo and assessed the potential correlation between the expression of $\mathrm{KDM} 4 \mathrm{~B}$ and $\mathrm{CRC}$ prognosis.
\end{abstract}

Results: We found that KDM4B promoted glucose uptake and ATP production by regulating the expression of GLUT1 via the AKT signaling pathway. KDM4B could interact with TRAF6 and promote TRAF6-mediated ubiquitination of AKT for AKT activation. Furthermore, we demonstrated that KDM4B was overexpressed in CRC specimens and high level of KDM4B was associated with a poor survival rate in CRC patients.

Conclusions: These findings reveal that KDM4B plays an important role in promoting CRC progression by enhancing glucose metabolism.

Keywords: KDM4B, GLUT1, AKT activation, TRAF6, Glucose metabolism

\section{Background}

Colorectal cancer (CRC) is one of the most common cancers in the world. It is the third most commonly diagnosed cancer in men and the second in women worldwide [1]. In Asian countries, the incidence of $\mathrm{CRC}$ has been rising rapidly [2]. However, the underlying molecular mechanisms responsible for CRC tumorigenesis are still not fully understood.

The metabolic properties of cancer cells are distinct from those of normal cells [3].The best characterized metabolic phenotype observed in tumor cells is the Warburg effect, which is a shift from ATP generation

\footnotetext{
* Correspondence: Iuoxl@tjh.tjmu.edu.cn

${ }^{+}$Haijie Li and Jingqin Lan contributed equally to this work.

Department of Gastrointestinal Cancer Research Institute, Tongji Hospital,

Tongji Medical College, Huazhong University of Science and Technology, Wuhan, Hubei, China
}

through oxidative phosphorylation to ATP generation through glycolysis, even in the presence of sufficient oxygen [4]. Although glycolysis can make ATP production more rapidly than oxidative phosphorylation, it is far less efficient in terms of ATP generated per unit of glucose consumed. This shift therefore demands that tumor cells implement an abnormally high rate of glucose uptake to meet the increased energy needs [5]. In the regulation of glucose uptake, the PI3K/AKT signaling plays a role of master regulator. PI3K/AKT signaling promotes both the expression of glucose transporter GLUT1 and the translocation of GLUT1 protein from the endo-membranes to the cell surface [6]. In addition, AKT potentiates the activity of the HK (Hexokinase), which phosphorylates glucose molecules, thus preventing their efflux back into the extracellular space [7]. 
KDM4B is an important member of the histone demethylase KDM4 family that is characterized by the catalytic Jumonji C domain. KDM4B is thought to regulate gene expression by demethylating tri-and di-methylated histone $\mathrm{H} 3$ at lysine 9 and lysine 36 [8]. Expression levels of KDM4B are notably upregulated in numerous cancers, including breast, prostate, bladder, ovarian, gastric and colorectal cancer [9-12]. It has been reported that KDM4B has a crucial role in human carcinogenesis by regulating cell cycle progression and apoptosis [13, 14]. KDM4B also plays an important role in cancer metastasis, DNA damage response and cell differentiation $[12,15,16]$. Recent study shows that KDM4B protects against obesity and metabolic dysfunction [17]. It is made us realized the significant role of KDM4B in metabolism. However, little is currently known about the role of KDM4B in glucose metabolism.

In the present study, we investigated the role of KDM4B in glucose metabolism. Our results showed that KDM4B promoted glucose uptake and ATP production by regulating the expression of GLUT1 via the AKT signaling pathway through the combination with TRAF6. Furthermore, we demonstrated that KDM4B was overexpressed in CRC specimens and high level of KDM4B was associated with a poor survival rate in CRC patients. Collectively, we reveal that KDM4B plays an important role in promoting CRC progression by enhancing glucose metabolism.

\section{Materials and methods}

\section{Cell lines and cell culture}

The human CRC cell lines LoVo, SW620, HCT116 and human embryonic kidney HEK293 T were purchased from the Cell Bank of Type Culture Collection of Chinese Academy of Sciences (Shanghai, China). LoVo, SW620 and $293 \mathrm{~T}$ cells were cultured at $37^{\circ} \mathrm{C}, 5 \% \mathrm{CO}_{2}$ in DMEM medium containing $10 \%$ fetal bovine serum (GIBICO, NY, USA). HCT116 cells were cultured in McCoy's $5 \mathrm{~A}$ medium containing $10 \%$ fetal bovine serum (GIBICO).

\section{Antibodies}

The following antibodies were used for Western blot analysis, Co-IP, immunofluorescence or immunohistochemistry assay: anti-KDM4B (2639, Cell Signaling Technology), anti-GLUT1(12,939, Cell Signaling Technology), anti-AKT (2920, Cell Signaling Technology), anti-Phosoho-AKT Ser473 (4060, Cell Signaling Technology), Phosoho-AKT Thr308 (4056, Cell Signaling Technology), anti-TRAF6 (8028, Cell Signaling Technology), anti-6X His tag(ab18184, Abcam), antiHA tag(ab9110, Abcam), anti-Flag tag(SAB4301135, Sigma-Aldrich), anti-TXNIP(14,715, Cell Signaling Technology).

\section{RNA interference and expression plasmid}

siRNA duplexes targeting the human $K D M 4 B$ gene were synthesized and purified by RiboBio (Ribobio, Guangzhou, China). siRNA duplexes with non-specific sequences were used as an siRNA-negative control. RNA oligonucleotides were transfected using Lipofectamine RNAiMAX Reagent (Invitrogen) and the expression levels of KDM4B were quantified $72 \mathrm{~h}$ after transfection. KDM4B siRNA was designed as follows: siKDM4B 1\# 5' - GCGCAGAAUCUACCAACUU-3', siKDM4B 2\# 5' CGGCCACAUUACCCUCCAA-3'.

cDNA constructs encoding KDM4B were cloned into the pcDNA3.1 expression vector and Flag expression vector using standard cloning methodology. TRAF6 with HA-tag and AKT with HA-tag was also constructed by our team in the same way. Ubiquitin with his-tag was bought from Biovector Science Lab (NTCC, Beijing, China). The myr-AKT plasmid was a generous gift from Dr. Hui Kuan Lin (Department of Cancer Biology, Wake Forest Baptist Medical Center, NC, USA).

Eukaryotic expression plasmids $(1 \mu \mathrm{g})$ were transfected into $293 \mathrm{~T}$ cells or CRC cells in 6-well plates using $10 \mathrm{ul}$ Lipofectamine 3000 (Invitrogen). Cells were harvested after $72 \mathrm{~h}$ for further analysis.

\section{Cell cycle analysis and BrdUrd incorporation analysis}

Cells were fixed in $80 \%$ ethanol overnight at $-20^{\circ} \mathrm{C}$, washed with phosphate-buffered saline, and then stained with propidium iodide and $100 \mu \mathrm{g} / \mathrm{ml}$ RNaseA. DNA content was measured by sorting the fluorescenceactivated cells on a Becton-Dickinson FACScan System (Franklin Lakes, NJ, USA). For the BrdUrd incorporation analysis, cells were incubated in BrdUrd medium at $10 \mu \mathrm{g} / \mathrm{ml}$ for $30-\mathrm{min}$. After aspirating the medium, the cells were immediately fixed for more than $8 \mathrm{~h}$ at $20^{\circ} \mathrm{C}$. After immunostaining using the BrdUrd antibody, the DNA synthesis rate was assessed by calculating the percentage of $\mathrm{BrdUrd}^{+}$cells out of the total cell count on BD FACScan System.

\section{Glucose uptake and ATP detection}

Intracellular glucose uptake was determined by 2deoxyglucose-6-phosphate (2DG6P), a fluorescentlytagged glucose derivative, using a Glucose Uptake Cell-Based Assay Kit (Promega, WI, USA) in accordance with the manufacturer's protocol. The cells were incubated with 2-deoxyglucose (2DG) for $10 \mathrm{~min}$ in 96-wells and the followed with the protocol to detect the luminescent signal that is proportional to the concentration of 2DG6P.

Cellular ATP levels were measured using a firefly luciferase-based Bioluminescence ATP assay kit (Beyotime, Jiangsu, China). Briefly, cells were lysed and centrifuged at $12,000 \mathrm{~g}$ for $5 \mathrm{~min}$ at $4{ }^{\circ} \mathrm{C}$. Then 100ul of each supernatant 
was mixed with 100ul of ATP detection solution. Luminance (RLU) was measured by a Luminometer.

\section{Immunofluorescence}

Cells were rinsed with PBS, fixed in 4\% paraformaldehyde for $10 \mathrm{~min}$ at room temperature, and permeabilized with $0.1 \%$ Triton X-100 for $10 \mathrm{~min}$. The cells were blocked with 2\% BSA-PBS at room temperature for 60 min, and incubated with primary antibodies at $4{ }^{\circ} \mathrm{C}$ overnight. After washing 3 times with PBS, cells were incubated with secondary antibodies for $60 \mathrm{~min}$ at $37^{\circ} \mathrm{C}$, and fluorescence at $488 \mathrm{~nm}$ (FITC) and $543 \mathrm{~nm}$ (TRITC) were detected using the LSM 710 confocal laser scanning microscope.

\section{Cell fractionation}

Cytosolic and membrane fractions were prepared using the Mem-PER Plus Membrane Protein Extraction Kit (Thermo Fisher) according to the manufacturers' standard procedures.

Briefly, cells were suspended in Permeabilization Buffer for $10 \mathrm{~min}$ at $4{ }^{\circ} \mathrm{C}$. The permeabilized cells were centrifuged at $16000 \mathrm{~g}$ for $15 \mathrm{~min}$ at $4{ }^{\circ} \mathrm{C}$. The resulting supernatant (cytosol) was collected and the sediment was resuspended by Solubilization Buffer. The lysate was again centrifuged at $16000 \mathrm{~g}$ for $15 \mathrm{~min}$ at $4{ }^{\circ} \mathrm{C}$ and the supernatant (membrane) was collected. The distribution of proteins in the cytosol and membrane fractions was analyzed by western blotting.

\section{Immunoprecipitation and immunoblotting}

Two hundred ninety-three $\mathrm{T}$ cells were cultured in DMEM medium containing 10\% FBS. Transfection was performed with Lipofectamine 3000 and RNAiMAX (Invitrogen, California, USA). At $24-48 \mathrm{~h}$ after transfection, cells were harvested and lysed in NP40 lysis buffer supplemented with protease inhibitor cocktail (Beyotime, Jiangsu, China). Immunoprecipitation and immunoblotting were performed as described in Luo Zhang's article [18]. The Gel-pro Analyzer 4.0 was used to quantify the bands in immunoblotting images.

To detect endogenous interaction between KDM4B and TRAF6, immunoprecipitation was performed with $2 \mu \mathrm{g}$ of agarose-conjugated anti-TRAF6 antibody. Cells were incubated with IGF-1 $(100 \mathrm{ng} / \mathrm{ml})$ for $0,15,30$ and $60 \mathrm{~min}$ and then collected for the immunoprecipitation.

\section{Lentiviral system}

The generation of stable expression cells was performed by lentiviral system. KDM4B was depleted in LoVo and HCT116 cells using a KDM4B lentiviral construct expressing KDM4B-targeted short hairpin RNA (shKDM4B, Genechem, Shanghai, China). Following infection with lentivirus, cells were expanded in medium containing puromycin $(1.5 \mu \mathrm{g} / \mathrm{ml})$, and screened for KDM4B knockdown by western blot analysis. Empty vector-infected cells (shControl) were used as a control. Myr-AKT stable overexpression cells and control cells were generated in the same way.

\section{In vivo tumorigenesis}

The study was approved by the Huazhong University of Science and Technology (HUST) Ethics Committee. All animal experiments were performed accordance with the animal study guidelines of HUST. Nude mice (nu/nu, female, 5 weeks old) were injected subcutaneously with CRC cells $\left(1 \times 10^{6}\right.$ cells $)$ stably expressing the control and shcontrol vector (shControl+Control group), myrAKT with shcontrol vector (shControl +AKT group), shKDM4B with control vector (shKDM4B + Control group) and shKDM4B with myr-AKT vector (shKDM4B + AKT group). Ten animals per group were used in each group. Tumor growth was monitored for 35 days. Tumor size was measured with a caliper, and tumor volume was determined with a standard formula, $\mathrm{L} \times \mathrm{W}^{2} \times 0.5$, where $\mathrm{L}$ is the longest diameter and $\mathrm{W}$ is the shortest diameter. The tumors were dissected and analyzed every 5 days. Mice were sacrificed by cervical dislocated at indicated time and the tumors were removed for analysis.

\section{Tissue microarray and immunohistochemistry}

A commercially available paraffin-embedded human CRC tissue array was bought form Shanghai Outdo Biotech, catalog no. HColA180Su10. Demographic and clinicopathological data including clinical staging (According to the AJCC staging system) and survival data were provided by the manufacturer. Immunohistochemistry was performed as described previously [9].

KDM4B immunostaining was evaluated based on scores representing the percentage of positively stained tumor cells and the staining intensity grade. KDM4B positive cells were counted in 3 different fields and photographed using an Olympus microscope. The immunoreactions were evaluated independently by two pathologists blinded to the clinicopathologic information to ensure proper tissue morphology. The percentages of positively stained tumor cells were scored according to the following scale: 0 (no positive cells), 1 ( $<25 \%$ positive cells), 2 ( $25-50 \%$ positive cells), 3 (50-75\% positive cells), and $4(>75 \%$ positive cells). The staining intensities were classified into the following four categories: 0 (no staining); 1 (weak staining); 2 (moderate staining), 3 (strong staining). The score for each tissue was calculated by multiplying the intensity index with the percentage scale. The median value of KDM4B scores was employed to determine the cutoff. Tumors with KDM4B scores lower or equal to the median were designated as 
"low expression", whereas those with scores higher than the median were designated as "high expression".

\section{Statistical analysis}

The results were analyzed using SPSS 19 (Chicago, IL, USA). The data were expressed at the mean \pm SD. KDM4B expression between tumor tissues and matched nontumor tissues was analyzed with the paired Student's $t$ test. The association between KDM4B expression and various clinicopathological parameters was evaluated with the $x^{2}$ test. The Cox proportional hazard regression model was used for univariate and multivariate analyses to determine the effects of the clinicopathological variables and KDM4B expression on the patient survival. Only variables with $p$ values $<0.05$ in univariate analysis were included in multivariate analysis. Survival curves were calculated using the KaplanMeier method. In all statistical tests, $p$ values $<0.05$ were deemed statistically significant.

\section{Results}

KDM4B is necessary for CRC cells proliferation and glucose metabolism

Initially, we performed functional analysis using CRC cell lines to elucidate whether KDM4B was involved in proliferation and affected glucose metabolism in CRC. To identify the roles of KDM4B in cell proliferation, we detected the cell-cycle progression and DNA synthesis in KDM4B-depressed cells. We found that KDM4B knockdown caused a significant decrease in the cell number at S-phase and incorporated BrdUrd, showing that the knockdown of KDM4B blocked the cell-cycle procession and inhibited the DNA synthesis (Fig. 1a and b). In the meantime, KDM4B overexpression promoted the DNA synthesis (Additional file 1: Figure S1A).

In order to fulfill the biosynthetic demands associated with rapid proliferation, cancer cells must increase the import of nutrients form the environment. The main nutrient that supports survival and biosynthesis in mammalian cells is glucose. So we detected the role of KDM4B in glucose uptake which was the initial step of glucose metabolism. Our results showed that intracellular glucose uptake was significantly decreased in KDM4Bdepressed cells and increased in KDM4B-overexpressed cells (Fig. 1c and Additional file 1: Figure S1B). Furthermore, KDM4B-depressed cells displayed a significant decrease of cellular ATP content while KDM4B-overexpressed cells displayed a significant increase (Fig. 1d). We also examined glucose-induced TXNIP expression, which is commonly used as an intracellular

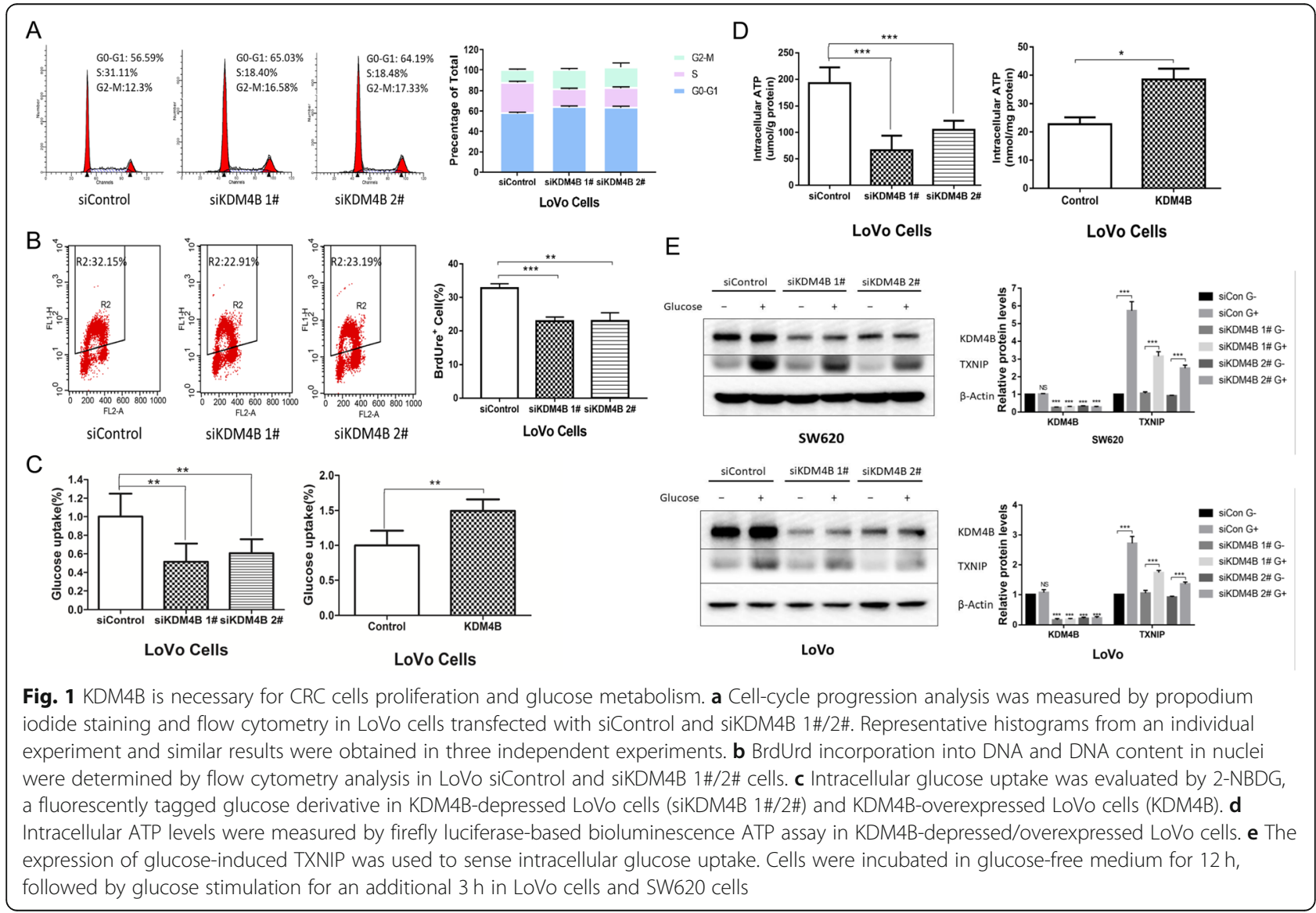


glucose sensor $[19,20]$. TXNIP was robustly induced in control cells following glucose stimulation. In contrast, the induction of TXNIP was suppressed in KDM4B-depressed cells, supporting the result that the depression of KDM4B inhibited intracellular glucose uptake in CRC cells (Fig. 1e). To further explore potential functions of KDM4B in CRC, gene expression profiling on GSE9348 from GEO database was employed. CRC tissues had more abnormal genes expression in Glucose transport pathway than normal tissues (Additional file 1: Figure S1C).

Overall, these results of functional analysis suggested that KDM4B promoted the proliferation and glucose metabolism of CRC cells through regulation glucose uptake, which encouraged us to further explore the significance of KDM4B in CRC metabolism.

\section{KDM4B promotes the expression of GLUT1 via the AKT} signaling pathway

Glucose uptake is controlled primarily by the glucose transporter family (GLUTs) which have 14 members [21]. GLUT1, the first member of the GLUT1 family to be identified, has been the most extensively studied. GLUT1 is overexpressed in many tumors, including hepatic, pancreatic, breast, colorectal and ovarian cancers [22-25]. GLUT1 is the main glucose transporter in most cell types, and unlike GLUT4, it appears to be regulated primarily through alterations in expression levels [26]. Since GLUT1 is essential for cancer cell glucose uptake, we then examined the effect of KDM4B on GLUT1 expression. As shown in Additional file 1: Figure S2 A-E, the expression of GLUT1 was suppressed in KDM4Bdepressed cells and was upregulated in KDM4Boverexpressed cells. These data indicate that KDM4B is involved in the regulation of GLUT1 expression.

In the regulation of GLUT1, the PI3K/AKT signaling plays a role of master regulator. To clarify the mechanism by which KDM4B regulate GLUT1, we further explored the role of KDM4B in AKT signaling pathways. The phosphorylation of AKT at Thr308 and Ser473 was inhibited in the KDM4B-depressed cells with the depressed GLUT1 (Fig. 2a and Additional file 1: Figure S3 A). Similarly, the phosphorylation of AKT at Thr308 and Ser473 was enhanced in KDM4B-overexpressed cells with the upregulated GLUT1 (Fig. 2b). To further test whether the regulation of GLUT1 by KDM4B depends on AKT or not, we enhanced the activation of AKT in KDM4B-depressed cells by transfected with a constitutively active mutant of AKT, myristoylated AKT (myr-AKT) which does not require its $\mathrm{PH}$ domain for plasma membrane recruitment, to detect the expression of GLUT1. We observed sustained phosphorylation of AKT efficiently rescued the expression of GLUT1 in KDM4B-depressed cells (Figure 2c and d). Similarly, the

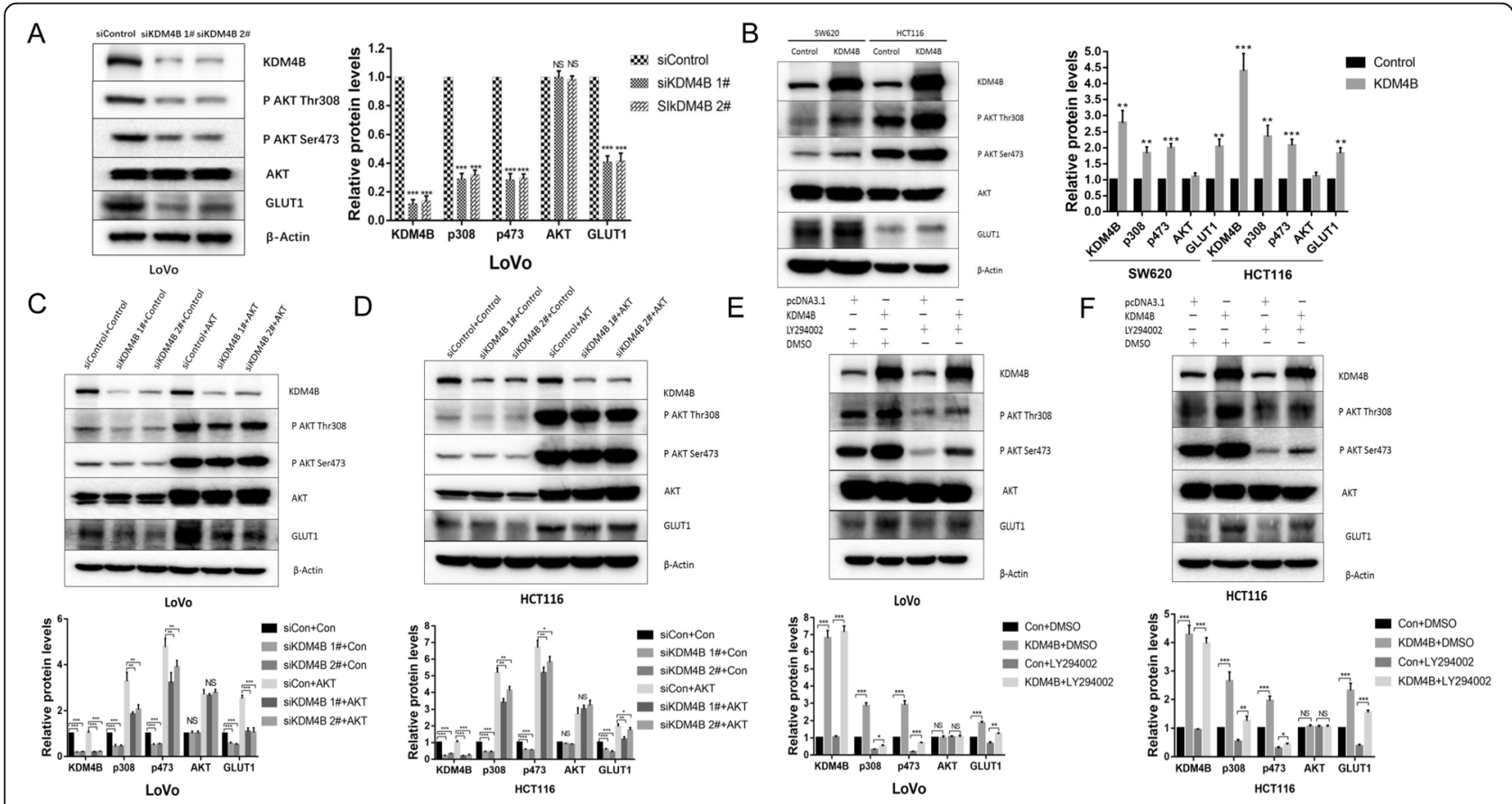

Fig. 2 KDM4B promotes the expression of GLUT1 via the AKT signaling pathway. $\mathbf{a}$ and $\mathbf{b}$ The phosphorylation of AKT at Thr 308 and Ser 473 and the expression of GLUT1 was detected in KDM4B-depressed(A)/overexpressed(B) CRC cells. $\mathbf{c}$ and $\mathbf{d}$ The expression of GLUT1 was detected in KDM4B-depressed LoVo/HCT116 cells transfected with myr-AKT. e and $\mathbf{f}$ The expression of GLUT1 was detected in KDM4B-overexpressed LoVo/ HCT116 cells incubated with PI3K inhibitor LY294002 
KDM4B-overexpressed cells with the PI3K inhibitor LY294002 efficiently reduced the expression of GLUT1(Fig. 2e and $\mathrm{f}$ ). These data suggest that the regulation of GLUT1 by KDM4B depends, at least partially, on AKT.

\section{KDM4B promotes membrane localization of AKT}

$\mathrm{AKT}$ is generally activated by stimulation of growth factor receptors on the surface in a multistep process that includes binding of AKT to PIP3, translocation of AKT from the cytosol to the membrane and phosphorylation of AKT at Thr308 and Ser473 by the upstream kinases PDK1 and mTORC2 [27, 28]. Consider that the binding to PIP3 and membrane translocation is the initial and essential step for AKT activation, we reasoned that a possible mechanism of KDM4B in AKT activation could be to promote the translocation of AKT from the cytosol to the plasma membrane. Our results showed that KDM4B knockdown inhibited the membrane phosphorylation of AKT (Fig. 3a and Additional file 1: Figure S3B). Consistently, immunofluorescence analysis showed that the localization of AKT proteins on the membranes were reduced in KDM4B knockdown cells than that of control cells (Fig. 3b). These results indicate that KDM4B promotes AKT activation through the regulation of AKT membrane recruitment.

\section{KDM4B interacts with TRAF6 and promotes TRAF6- mediated ubiquitination of AKT}

Protein ubiquitination through Lys48(K48) of the ubiquitin chain generally targets proteins for degradation, whereas ubiquitination through K63 plays a critical role in signaling activation and protein trafficking $[29,30]$. The K63 chain ubiquitination of AKT contributes to the enhancement of AKT membrane localization and phosphorylation [31]. As KDM4B promotes AKT membrane recruitment, we analyzed whether KDM4B regulated the ubiquitination of AKT. It has been proved that ubiquitination occurred through K63 but not through K48 in the absence of proteasome inhibitor MG132 [31]. The result showed that KDM4B knockdown inhibited the ubiquitination of AKT without MG132 (Fig. 3c).

It has been proved that the ubiquitin ligase TRAF6 promotes the K63-linked ubiquitination of AKT and is required for plasma membrane translocation of AKT [31]. As KDM4B promoted AKT membrane recruitment, we hypothesize that KDM4B may interact with TRAF6 to promote its effect on AKT. We observed that

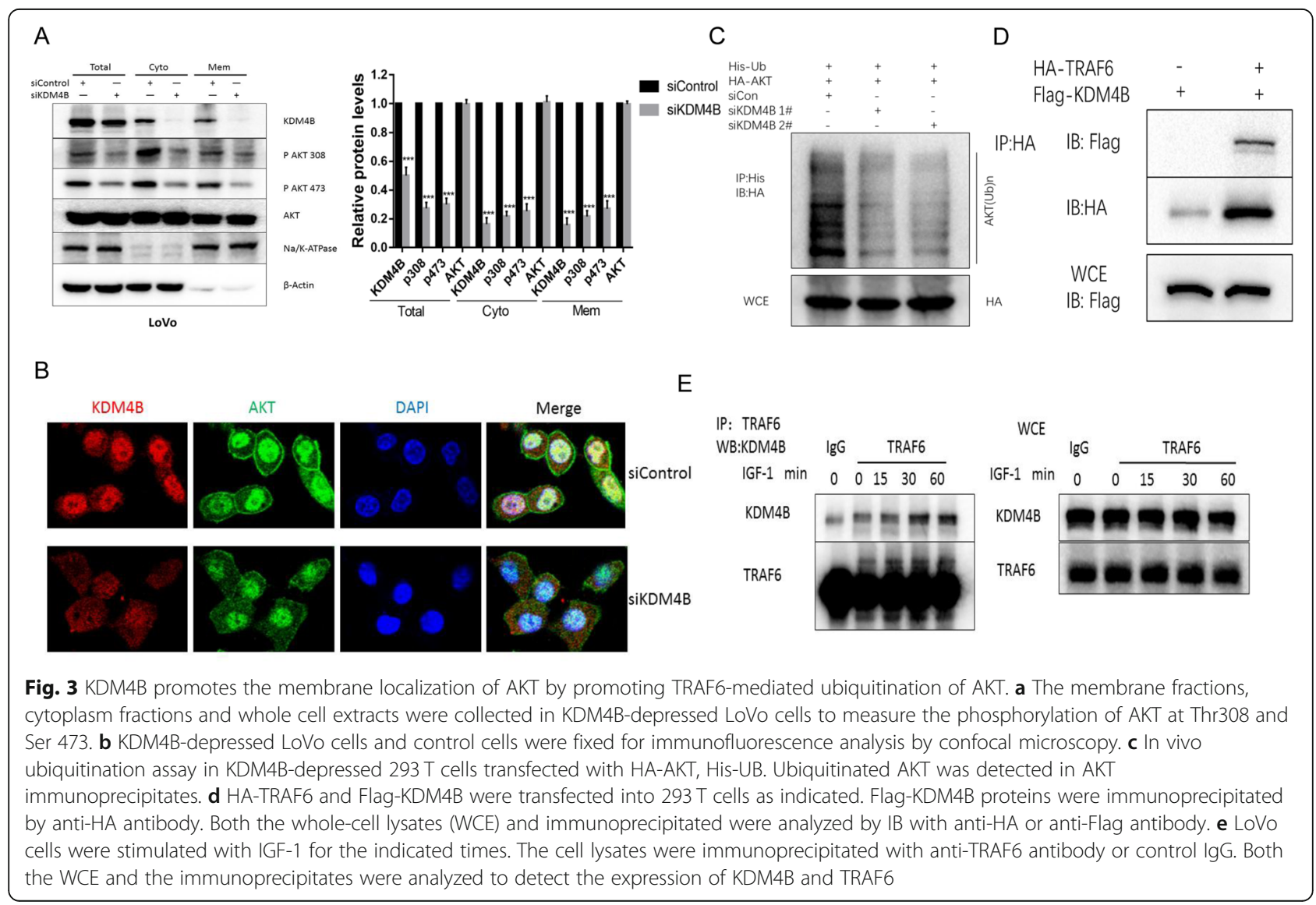


KDM4B interacted with TRAF6 upon IGF-1 stimulation (Fig. 3d and e). These results indicate that KDM4B interacts with TRAF6 and promotes TRAF6-mediated AKT activation.

\section{KDM4B promotes cells proliferation and glucose metabolism in a partly AKT-dependent manner}

To identify potential role of AKT in the regulation of KDM4B in cells proliferation and glucose metabolism, we detected the functional implication including glucose uptake, cell-cycle progression, DNA synthesis and clone formation in KDM4B-depressed cells with enhanced AKT activity. We observed that the enhanced AKT activity can partly rescue the glucose uptake ability depressed by KDM4B knockdown (Fig. 4a). Similarly, enhanced AKT activity can partly rescue the cell-cycle procession (Fig. 4b), DNA synthesis (Fig. 4c) and clone formation (Fig. 4d) blocked by KDM4B knockdown. To make our results more convincing, we detected the effect of AKT inhibition on cells proliferation and glycometabolism in KDM4B-overexpressed cells. We found that AKT inhibition could partly depress the enhancement of DNA synthesis (Additional file 1: Figure S3 C) and glucose uptake (Additional file 1: Figure S3 D) by KDM4B-overexpressed. These results indicate that
KDM4B promotes cells proliferation and glucose metabolism in a partly AKT-dependent manner.

\section{AKT inhibits KDM4B-depressed induction of tumor growth suppression in mouse xenograft models}

After confirming the mechanism that KDM4B promotes the expression of GLUT1 via the AKT signaling pathway in colorectal cells, we investigated whether AKT could rescue the KDM4B depress-induced suppression of tumor growth in mouse xenograft models. To test this, we generated colorectal cell lines expressing KDM4B shRNA, myr-AKT plasmid alone or simultaneously expressing KDM4B shRNA and myr-AKT plasmid by lentivirus. We found that KDM4B knockdown significantly suppressed tumor growth, both in the tumor volume and weight (Fig. 5a-c). But the inhibition of tumor growth by KDM4B knockdown was partially rescued when AKT was constitutively active in the meantime in vivo (Fig. 5a-c). In support of this, we detected lower Ki-67 staining in KDM4B knockdown tumor tissues and stronger Ki-67 staining in simultaneous KDM4B knockdown and AKT constitutively activation tumor tissues by the immunohistochemistry analysis (Fig. 5d). Collectively, these results demonstrate that the loss of KDM4B in CRC cells results in the suppression

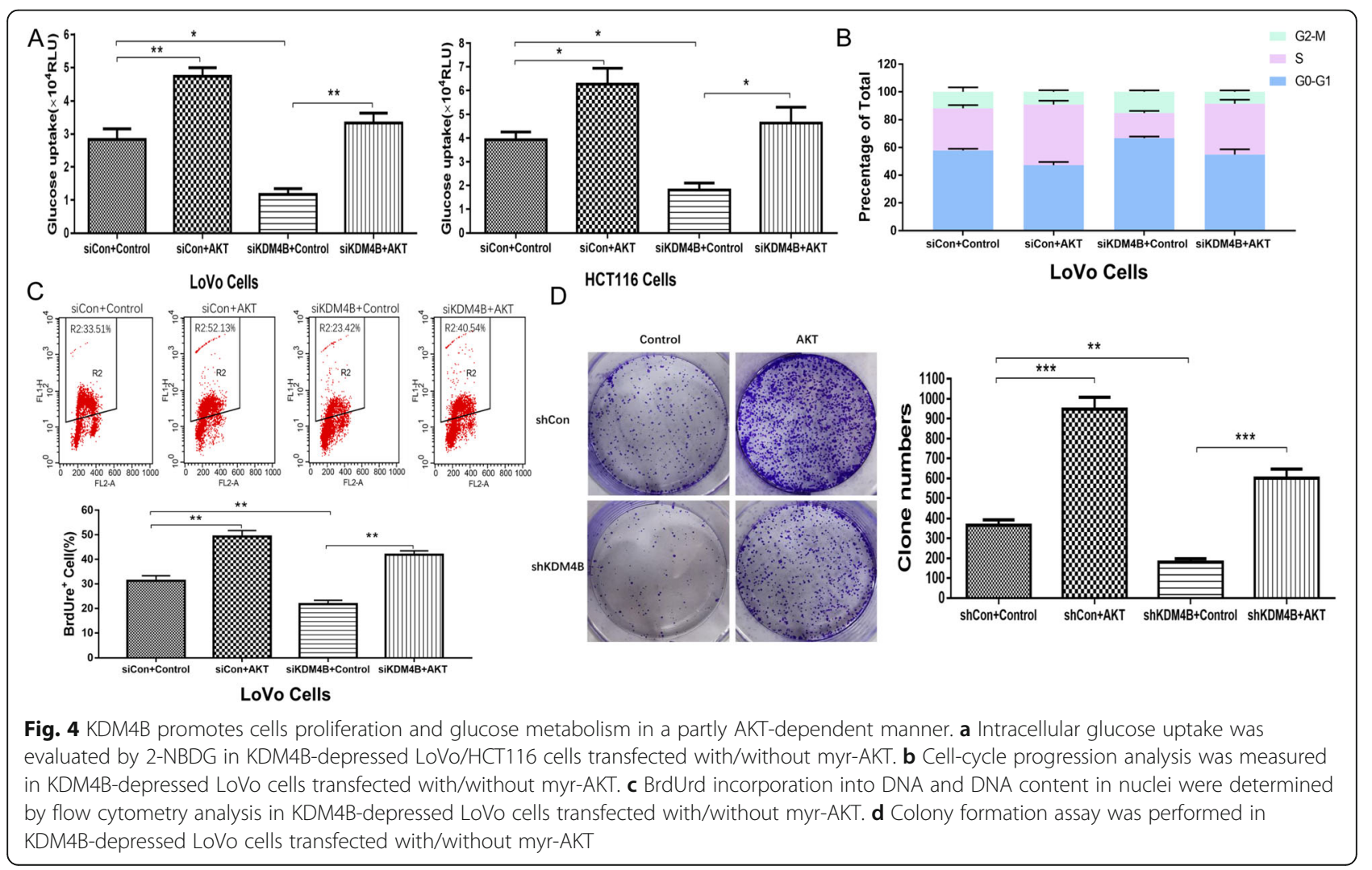




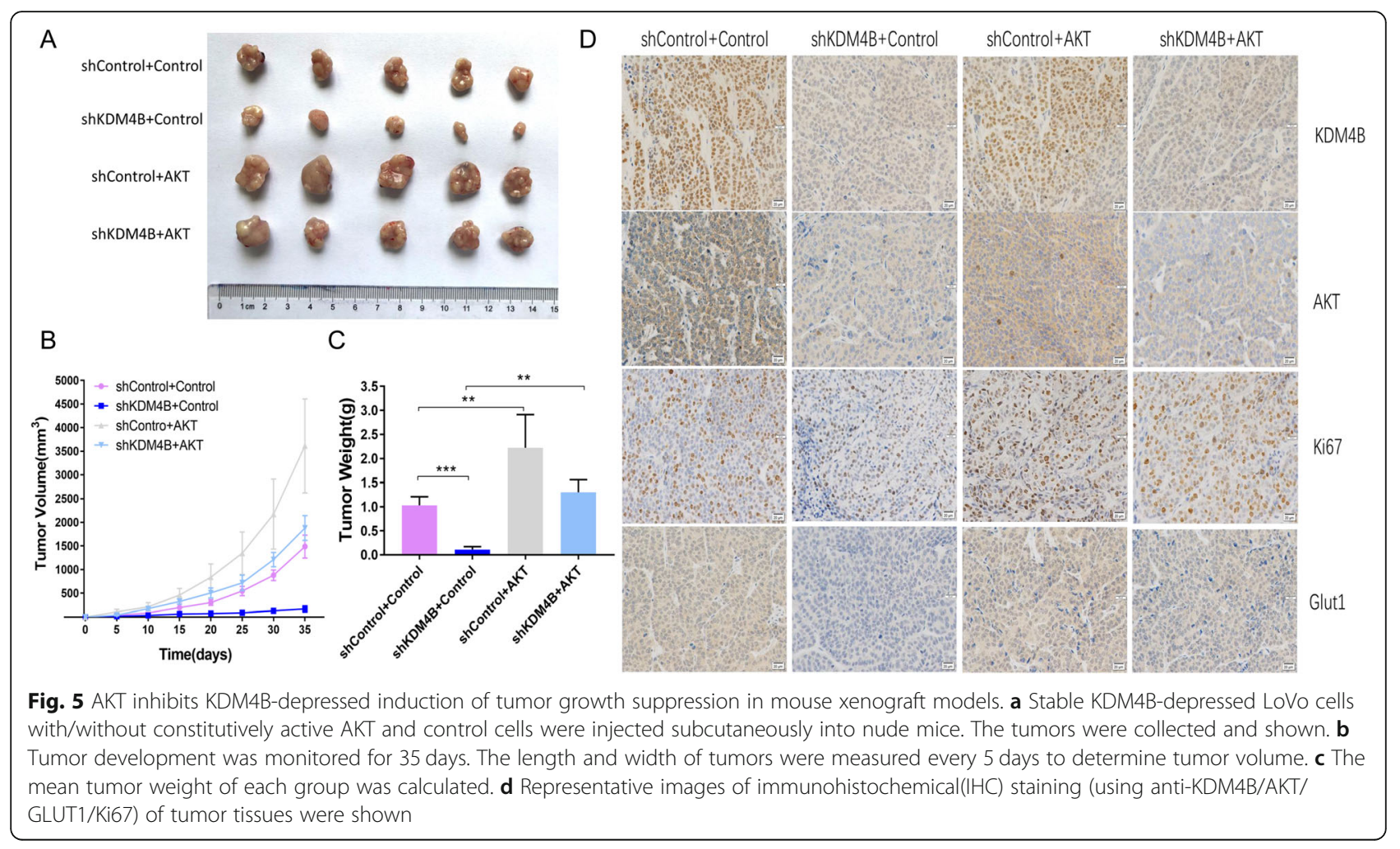

A

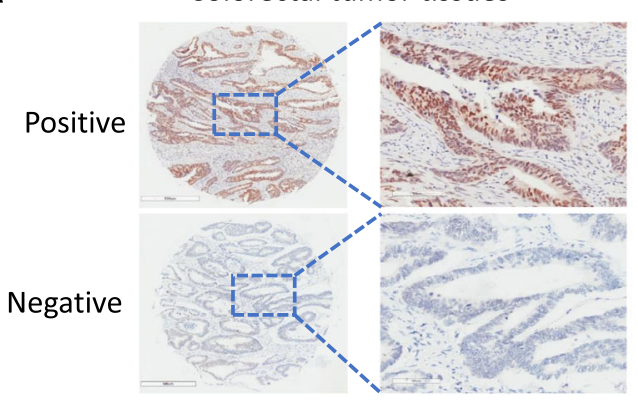

B

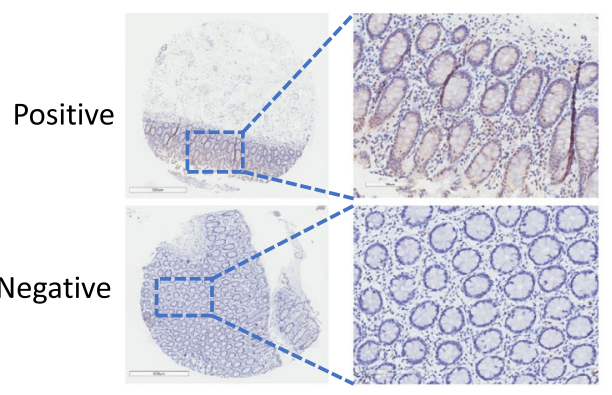

C

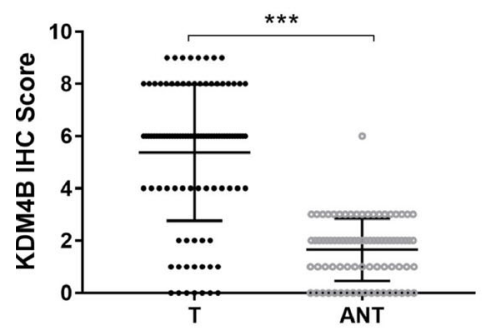

D

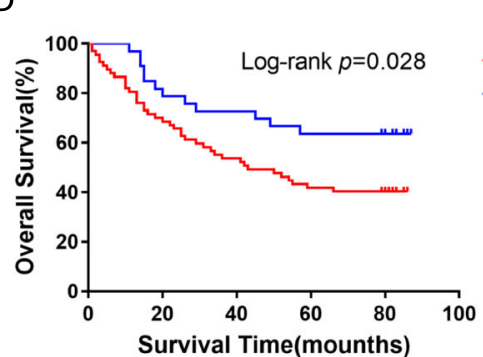

$\mathrm{E}$

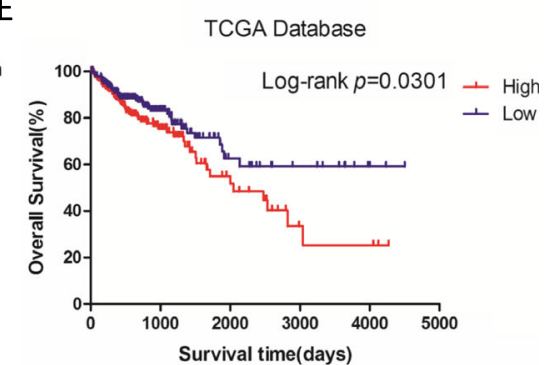

Fig. $6 \mathrm{KDM} 4 \mathrm{~B}$ is frequently up-regulated in colorectal tumor tissues and positive expression is correlated with an unfavorable prognosis. a and $\mathbf{b}$ Representative results of immunohistochemical staining for KDM4B expression in colorectal cancer tissues (a) and adjacent nontumor tissues (b). c Dot plots showing immunohistochemical scores and mean \pm SD of KDM4B in colorectal tumor and NT tissues. $\mathbf{d}$ Kaplan-Meier survival curves of patients with colorectal cancer in the tissue microarray $(n=80)$ segregate into high- and low-KDM4B expression groups. e Kaplan-Meier survival curves of patients with colorectal cancer in TCGA database segregate into high- and low-KDM4B expression groups 
of tumor growth and the suppression can be partly rescued by constitutively active AKT.

\section{KDM4B is frequently up-regulated in colorectal tumor tissues and positive expression is correlated with an unfavorable prognosis}

Given the striking effect of KDM4B on tumor growth, we then questioned whether there was an association between KDM4B expression and clinical prognosis in patients with colorectal cancer. We next evaluated the expression of KDM4B by immunohistochemical staining on a tissue microarray containing 180 spots, of which 160 were paired CRC tissue and corresponding adjacent nonneoplastic mucosa tissue from 80 patients, and the remaining spots were CRC tissue form 20 other patients. Representative slides of CRC tissues and corresponding adjacent nonneoplastic mucosa tissue are shown in Fig. $6 \mathrm{a}$ and b. Notably, the expression of KDM4B was up-regulated in tumor tissues than adjacent nonneoplastic mucosa tissue (Fig. 6c). Furthermore, we noted the existence of a significant correlation between tumor KDM4B expression levels and pathologic $\mathrm{T}$ stage, as well as clinical stage, who underwent pre-therapy surgery (Table 1). Univariate Cox regression analyses indicated

Table 1 Correlation between KDM4B expression levels and clinicopathological parameters in 100 cases of colon canser

\begin{tabular}{|c|c|c|c|c|}
\hline Variable & $\mathrm{N}$ & High KDM4B & Low KDM4B & $P$ Value \\
\hline \multicolumn{5}{|l|}{ Gender } \\
\hline Male & 59 & 39 & 20 & \\
\hline Female & 41 & 28 & 13 & $>0.9999$ \\
\hline \multicolumn{5}{|l|}{ Age } \\
\hline$<60$ & 24 & 18 & 6 & \\
\hline$\geq 60$ & 76 & 49 & 27 & 0.4567 \\
\hline \multicolumn{5}{|c|}{ Tumor size (cm) } \\
\hline$<5$ & 34 & 24 & 10 & \\
\hline$\geq 5$ & 66 & 43 & 13 & 0.6574 \\
\hline \multicolumn{5}{|l|}{ T-stage } \\
\hline $1 / 2$ & 3 & 0 & 3 & \\
\hline $3 / 4$ & 97 & 67 & 30 & 0.0337 \\
\hline \multicolumn{5}{|l|}{$\mathrm{N}$-stage } \\
\hline NO & 53 & 32 & 21 & \\
\hline $\mathrm{N} 1$ & 35 & 24 & 11 & \\
\hline N2 & 12 & 11 & 1 & 0.1112 \\
\hline \multicolumn{5}{|l|}{ M-stage } \\
\hline MO & 95 & 63 & 32 & \\
\hline M1 & 5 & 4 & 1 & $>0.9999$ \\
\hline \multicolumn{5}{|c|}{ Clinical Stage } \\
\hline$|/| \mid$ & 52 & 29 & 23 & \\
\hline III/IV & 48 & 38 & 10 & 0.0188 \\
\hline
\end{tabular}

that KDM4B expression, lymph node metastasis ( $\mathrm{N}$ stage), distant metastasis ( $\mathrm{M}$ stage) and clinical stage were significantly associate with patient survival (Table 2). Furthermore, a multivariate Cox regression analysis further confirmed KDM4B expression, lymph node metastasis ( $\mathrm{N}$ stage) and distant metastasis ( $\mathrm{M}$ stage) as independent predictors of shorter OS (Table 2). More importantly, Kaplan-Meier analysis showed high KDM4B expression was significantly correlated with shorter survival (Logrank, $p=0.028$, Fig. $6 \mathrm{~d}$ ). Analysis of the gene expression with survival data from the TCGA database also showed similar results (Log-rank, $p=0.0301$, Fig. 6e). Taken together, all these findings suggested that high expression of KDM4B indicated unfavorable prognosis.

\section{Discussion}

In this study, we wished to elucidate the specific role of lysine demethylase KDM4B on CRC growth and glucose metabolism. Our findings suggest that KDM4B facilitates CRC growth and glucose metabolism by stimulating AKT activation. KDM4B can interact with TRAF6 and promote TRAF6-mediated the K63 chain ubiquitination of AKT which contributes to the enhancement of AKT membrane localization and phosphorylation.

The oncogenic activities of KDM4B have been extensively investigated in multiple cancers, including breast, prostate, bladder, ovarian, gastric and colorectal cancer. KDM4B can promote tumor growth, apoptosis, metastasis and autophagy [9-12, 32]. All these functions cannot be separated from the support of energy metabolism. Compared to normal cells, cancer cells rewire their metabolism to differently utilize glucose for their energy needs. In cancer cells, glucose is converted to energy primarily by aerobic glycolysis [33]. This faster conversion of glucose to energy is required to meet the needs of rapidly growing cancer cells.

Recently, KDM3A, a demethylase that removes methyl form histone lysine $\mathrm{H} 3 \mathrm{~K} 9$, has been reported to promote urinary bladder cancer progression by enhancing glycolysis through coactivation of hypoxia inducible factor $1 \alpha$ [34]. KDM5B, another demethylase, has been reported to participate in the regulation of islet function and glucose homeostasis [35]. We also notice that the loss of KDM4B has been reported to result in metabolic dysfunction [17]. Consider the oncogenic role of KDM4B in multiple cancers, we wonder whether KDM4B can participate in glucose metabolism and the mechanism should be further investigated. In the present study, we identify a role for KDMB in promoting glucose uptake from extracellular environment by stimulating TRAF6-mediated AKT activation.

In previous studies, the role of KDM4 was mostly focused on the histone posttranslational modifications that regulate chromatin structure in nucleus for the past 
Table 2 Univariable and multivariable analyses of overall survival (OS) and clinicopathologic variables in 100 cases of colon cancer

\begin{tabular}{|c|c|c|c|c|c|}
\hline Variable & No. & $\mathrm{HR}(95 \% \mathrm{Cl})$ & $p$ value & HR $(95 \% \mathrm{Cl})$ & $p$ value \\
\hline \multicolumn{6}{|l|}{ Gender } \\
\hline Male & 59 & $1.262(0.721-2.207)$ & 0.415 & NA & \\
\hline Female & 41 & & & & \\
\hline \multicolumn{6}{|l|}{ Age } \\
\hline$<60$ & 24 & $1.449(0.727-2.889)$ & 0.292 & NA & \\
\hline$\geq 60$ & 76 & & & & \\
\hline \multicolumn{6}{|c|}{ Tumor size(cm) } \\
\hline$<5$ & 34 & $1.43(0.801-2.554)$ & 0.226 & NA & \\
\hline$\geq 5$ & 66 & & & & \\
\hline \multicolumn{6}{|l|}{ T-stage } \\
\hline $1 / 2$ & 3 & $1.262(0.761-2.092)$ & 0.367 & NA & \\
\hline $3 / 4$ & 97 & & & & \\
\hline \multicolumn{6}{|l|}{ N-stage } \\
\hline No & 53 & 1.819 (1.219-2.712) & 0.003 & $2.438(1.258-4.724)$ & 0.008 \\
\hline N1 & 35 & & & & \\
\hline N2 & 12 & & & & \\
\hline \multicolumn{6}{|l|}{ M-stage } \\
\hline MO & 95 & $5.142(1.984-13.326)$ & 0.001 & $9.299(2.143-40.355)$ & 0.003 \\
\hline M1 & 5 & & & & \\
\hline \multicolumn{6}{|c|}{ Clinical Stage } \\
\hline$|/| \mid$ & 52 & $1.893(1.206-2.973)$ & 0.006 & $0.568(0.249-1.297)$ & 0.179 \\
\hline III/IV & 48 & & & & \\
\hline \multicolumn{6}{|c|}{ KDM4B expression } \\
\hline Low & 33 & $2.025(1.062-3.863)$ & 0.032 & $1.862(0.964-3.597)$ & \\
\hline High & 67 & & & & 0.044 \\
\hline
\end{tabular}

studies. The posttranslational modifications include the demethylation of key lysine residues on the $\mathrm{N}$-terminal tail of histone $\mathrm{H} 3$ such as H3K9me3, H3K27me3 and H3K36me3, which are involved in promoter silencing and activating transcription [8]. But it is still unclear whether the KDM4B has some role in non-histone modification. In the meantime, some studies have previously demonstrated that KDM4B is also localized in cytoplasm [36]. The role of KDM4B in cytoplasm is also need our investigate. In our study, we demonstrate that the intracellular localization of KDM4B is both in cytoplasm and nucleus by immunofluorescence analysis and the KDM4B in cytoplasm could interact with TRAF6 and promote TRAF6-mediated AKT activation (Fig. 3). These results broaden our understanding the role of KDM4B in cell signaling.

Glucose transport is located at a "gate" position in the glycolytic flux to respond to various stimuli crucial for the Warburg effect, which is directly mediated by GLUT proteins. Our results identify that KDMB plays an important role in glucose uptake and regulates the expression of GLUT1, the main glucose transporter in CRC cells. The result is consisted with the previous study. In LN Fu et al work, they reported that knockdown of KDM4B could transcriptionally inhibit GLUT1 expression through increasing H3K9 tri-methylation levels on GLUT1 promoter [37]. In our study, we present a novel mechanism which KDM4B participate in glucose metabolism by the regulation of GLUT1. Our study shows that KDM4B can interact with TRAF6 in cytoplasm and promote TRAF6-mediated K63linked ubiquitination of AKT. The ubiquitination of $\mathrm{AKT}$ is required for activation of AKT and plays an important role in the regulation of GLUT1 expression (Fig. 7). This novel mechanism enables us to fully understand the important regulatory role of KDM4B in glucose metabolism.

PI3K/AKT signal pathway plays the central role in energy metabolism and coordinates diverse biological processes ranging from cell growth and differentiation to carcinogenesis [38, 39]. Our experiments reveal that KDM4B can acts closely upstream of or directly at AKT 


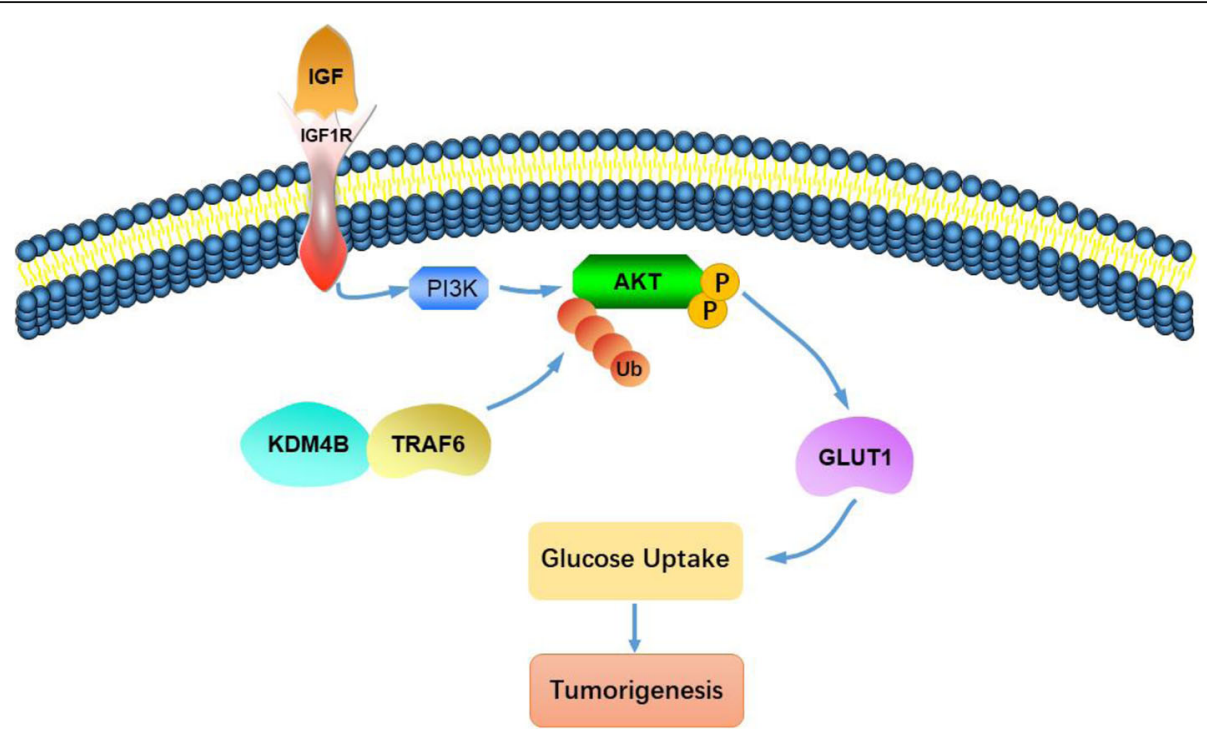

Fig. 7 The proposed working model of KDM4B in regulating the GLUT1 expression by the activation of AKT

phosphorylation (Fig. 2). The membrane translocation of AKT from the cytosol to the membrane is the initial and essential step for AKT activation [40]. Our results indicate that the knockdown of KDM4B inhibits AKT activation through impairing $\mathrm{AKT}$ membrane recruitment (Fig. 3). While PI(3,4,5)P3 formation induced by PI3K is clearly critical for membrane recruitment and activation of AKT upon growth factor stimulation, recent studies have revealed that K63-linked ubiquitination of AKT induced by growth factor is also a prerequisite for these processes [41]. The ubiquitin E3 ligase TRAF6 promotes the K63-linked ubiquitination of AKT and is required for plasma membrane translocation of AKT [31]. In our study, we have found that knockdown of KDM4B impaired the K63-linked ubiquitination and KDM4B interacted with TRAF6 upon IGF-1 stimulation (Fig. 3).

Much more attention has been focused on the role of lysine methylase and de methylase on non-histone proteins. In $\mathrm{GH}$ Wang et al work, they identified SETDB1, a lysine methylation, as an AKT-interacting protein that methylates AKT at K64 to elicit AKT ubiquitination. SETDB1-mediated K64 methylation of AKT serves as a scaffold to recruit histone demethylase KDM4A, which brings AKT's E3 ligases (TRAF6 and Skp2-SCF) to the AKT complex, thereby promoting AKT K63-linked ubiquitination, cell membrane recruitment and activation as well as tumorigenesis [42]. In M Dasgupta et al work, they reported STAT3-driven transcription depends upon the demethylation of K49 by EZH2 [43]. Our study also showed KDM4B could interact with TRAF6 in cytoplasm and promote TRAF6mediated K63-linked ubiquitination of AKT. The role of KDM4B on non-histone proteins should be more investigated in our further study.
Studies aiming to develop effective diagnostic and prognostic biomarker for cancer are urgently needed. In this study, we found that KDM4B is frequently upregulated in colorectal cancer. Our findings strongly suggest that KDM4B may be a useful diagnostic biomarker for colorectal cancer. More importantly, higher KDM4B expression in primary tumors is significantly correlated with unfavorable tumor stages and shorter survival in patients with colorectal cancer (Fig. 6). There findings therefore underpin an oncogenic role for KDM4B in colorectal cancer. The reason why KDM4B is overexpressed in colorectal cancer remains to be explored.

\section{Conclusions}

In summary, our study demonstrates that KDM4B facilitates colorectal cancer growth and glucose metabolism by stimulating TRAF6-mediated AKT activation, implicating that KDM4B is a potential molecular target for colorectal cancer treatment.

\section{Supplementary information}

Supplementary information accompanies this paper at https://doi.org/10. 1186/s13046-020-1522-3.
Additional file 1 : Figure S1. A BrdUrd incorporation into DNA and DNA content in nuclei were determined by flow cytometry analysis in KDM4B-overexpressed LoVo cells. B Intracellular glucose uptake was eval- uated by 2-NBDG, a fluorescently tagged glucose derivative in KDM4B- depressed SW620 cells (siKDM4B 1\#/2\#) and KDM4B-overexpressed SW620 cells (KDM4B). C GSE9348 RNA sequencing from GEO database was performed in CRC tissues and normal tissues. Pathway enrichment analysis revealed that Glucose transport pathway was more correlated in CRC tissues. Figure S2. A The expression of GLUT1 and GLUT2 in KDM4B-depressed LoVo cells in mRNA levels. B and C The expression of GLUT1 was detected in KDM4B-depressed LoVo/SW620 cells transfected with siControl and siKDM4B 1\#/2\#. D and E The expression of GLUT1 was detected in KDM4B-overexpressed LoVo/HCT116 cells transfected with 
KDM4B plasmid. Figure S3. A The phosphorylation of AKT at Thr308 and Ser 473 and the expression of GLUT1 was detected in KDM4B-depressed HCT116 cells. B The membrane fractions, cytoplasm fractions and whole cell extracts were collected in KDM4B-depressed SW620 cells to measure the phosphorylation of AKT at Thr308 and Ser 473. C BrdUrd incorporation into DNA and DNA content in nuclei were determined by flow cytometry analysis in KDM4B-overexpressed LoVo cells treated with/without LY294002. D Intracellular glucose uptake was evaluated by 2-NBDG in KDM4B-overexpressed LoVo cells treated with/without LY294002.

\section{Abbreviations}

BrdUrd: 5-bromo-2'-deoxyuridine; CRC: Colorectal cancer; GLUT1: Glucose transporter 1; IGF-1: Insulin like growth factor 1; myr-AKT: myristoylated AKT; PDK1: Pyruvate dehydrogenase kinase 1; TRAF6: TNF receptor associated factor 6; TXNIP: Thioredoxin interacting protein

\section{Acknowledgements}

The authors thank Tongji hospital for great help in providing tumor tissues.

\section{Authors' contributions}

$\mathrm{XL}$ conceived the project, oversaw research, and wrote the manuscript. $\mathrm{XL}$, $H \mathrm{~L}$, and $\mathrm{JL}$ designed the experiments, analyzed and interpreted the data and made the figures. $\mathrm{HI}, \mathrm{JL}, \mathrm{GW}, \mathrm{KG}, \mathrm{CH}$ and $\mathrm{XL}$ performed the experiments, analyzed and interpreted the data. $\mathrm{JH}$ and $\mathrm{ZC}$ helped conceived the project and interpret the data. All authors read and approved the final manuscript.

\section{Funding}

This work was supported by the National Natural Science Foundation of China (No. 81272278) and the Science and Technology Program of Hubei Province (No. 2019CFB664).

\section{Availability of data and materials}

All of the data and material in this paper are available when requested.

\section{Ethics approval and consent to participate}

This study was approved by the Clinical Research Ethics Committee of Tongji Hospital. All patients were fully informed of the experimental procedures before tissue acquisition. All animal experimental procedures were performed in compliance with the institutional ethical requirements and were approved by the Tongji Hospital Committee for the Use and Care of Animals.

\section{Consent for publication}

Not applicable.

\section{Competing interests}

The authors declare that they have no competing interests.

\section{Received: 27 August 2019 Accepted: 7 January 2020}

\section{Published online: 13 January 2020}

\section{References}

1. Jemal A, Bray F, Center MM, Ferlay J, Ward E, Forman D. Global cancer statistics. CA Cancer J Clin. 2011;61(2):69-90.

2. Sung JJ, Ng SC, Chan FK, Chiu HM, Kim HS, Matsuda T, Ng SS, Lau JY, Zheng S, Adler S, et al. An updated Asia Pacific consensus recommendations on colorectal cancer screening. Gut. 2015;64(1):121-32.

3. Annibaldi A, Widmann C. Glucose metabolism in cancer cells. Curr Opin Clin Nutr Metab Care. 2010;13(4):466-70.

4. Warburg O. On the origin of cancer cells. Science. 1956;123(3191):309-14.

5. Cairns RA, Harris IS, Mak TW. Regulation of cancer cell metabolism. Nat Rev Cancer. 2011;11(2):85-95.

6. Wieman HL, Wofford JA, Rathmell JC. Cytokine stimulation promotes glucose uptake via phosphatidylinositol-3 kinase/Akt regulation of Glut1 activity and trafficking. Mol Biol Cell. 2007;18(4):1437-46.

7. Deprez J, Vertommen D, Alessi DR, Hue L, Rider MH. Phosphorylation and activation of heart 6-phosphofructo-2-kinase by protein kinase B and other protein kinases of the insulin signaling cascades. J Biol Chem. 1997;272(28): 17269-75.
8. Berry WL, Janknecht R. KDM4/JMJD2 histone demethylases: epigenetic regulators in cancer cells. Cancer Res. 2013;73(10):2936-42.

9. Li H, Yang X, Wang G, Li X, Tao D, Hu J, Luo X. KDM4B plays an important role in mitochondrial apoptosis by upregulating HAX1 expression in colorectal cancer. Oncotarget. 2016;7(36):57866-77.

10. Wilson C, Qiu L, Hong Y, Karnik T, Tadros G, Mau B, Ma T, Mu Y, New J, Louie RJ, et al. The histone demethylase KDM4B regulates peritoneal seeding of ovarian cancer. Oncogene. 2017;36(18):2565-76.

11. Shi L, Sun L, Li Q, Liang J, Yu W, Yi X, Yang X, Li Y, Han X, Zhang Y, et al. Histone demethylase JMJD2B coordinates H3K4/H3K9 methylation and promotes hormonally responsive breast carcinogenesis. Proc Natl Acad Sci U S A. 2011;108(18):7541-6.

12. Zhao L, Li W, Zang W, Liu Z, Xu X, Yu H, Yang Q, Jia J. JMJD2B promotes epithelial-mesenchymal transition by cooperating with beta-catenin and enhances gastric cancer metastasis. Clin Cancer Res. 2013;19(23):6419-29.

13. Toyokawa G, Cho HS, Iwai Y, Yoshimatsu M, Takawa M, Hayami S, Maejima K, Shimizu N, Tanaka H, Tsunoda T, et al. The histone demethylase JMJD2B plays an essential role in human carcinogenesis through positive regulation of cyclin-dependent kinase 6. Cancer Prev Res (Phila). 2011;4(12):2051-61.

14. Zhu S, Li Y, Zhao L, Hou P, Shangguan C, Yao R, Zhang W, Zhang Y, Tan J, Huang B, et al. TSA-induced JMJD2B downregulation is associated with cyclin B1-dependent survivin degradation and apoptosis in LNCap cells. J Cell Biochem. 2012;113(7):2375-82.

15. Young LC, McDonald DW, Hendzel MJ. Kdm4b histone demethylase is a DNA damage response protein and confers a survival advantage following gamma-irradiation. J Biol Chem. 2013;288(29):21376-88.

16. Lee HL, Yu B, Deng P, Wang CY, Hong C. Transforming growth factor-betainduced KDM4B promotes Chondrogenic differentiation of human Mesenchymal stem cells. Stem Cells. 2016;34(3):711-9.

17. Cheng Y, Yuan Q, Vergnes L, Rong X, Youn JY, Li J, Yu Y, Liu W, Cai H, Lin JD, et al. KDM4B protects against obesity and metabolic dysfunction. Proc Natl Acad Sci U S A. 2018;115(24):E5566-E75.

18. Zhang L, Wang Y, Xiao F, Wang S, Xing G, Li Y, Yin X, Lu K, Wei R, Fan J, et al. CKIP-1 regulates macrophage proliferation by inhibiting TRAF6mediated Akt activation. Cell Res. 2014;24(6):742-61.

19. Muoio DM. TXNIP links redox circuitry to glucose control. Cell Metab. 2007; 5(6):412-4.

20. Stoltzman CA, Peterson CW, Breen KT, Muoio DM, Billin AN, Ayer DE. Glucose sensing by MondoA:mlx complexes: a role for hexokinases and direct regulation of thioredoxin-interacting protein expression. Proc Natl Acad Sci U S A. 2008;105(19):6912-7.

21. Thorens B, Mueckler M. Glucose transporters in the 21st century. Am J Physiol Endocrinol Metab. 2010;298(2):E141-5.

22. Nishioka $T$, Oda $Y$, Seino $Y$, Yamamoto $T$, Inagaki $N$, Yano $H$, Imura $H$, Shigemoto R, Kikuchi H. Distribution of the glucose transporters in human brain tumors. Cancer Res. 1992;52(14):3972-9.

23. Haber RS, Rathan A, Weiser KR, Pritsker A, Itzkowitz SH, Bodian C, Slater G, Weiss A, Burstein DE. GLUT1 glucose transporter expression in colorectal carcinoma: a marker for poor prognosis. Cancer. 1998;83(1):34-40.

24. Wang BY, Kalir T, Sabo E, Sherman DE, Cohen C, Burstein DE. Immunohistochemical staining of GLUT1 in benign, hyperplastic, and malignant endometrial epithelia. Cancer. 2000;88(12):2774-81.

25. Cantuaria G, Fagotti A, Ferrandina G, Magalhaes A, Nadji M, Angioli R, Penalver M, Mancuso S, Scambia G. GLUT-1 expression in ovarian carcinoma: association with survival and response to chemotherapy. Cancer. 2001;92(5):1144-50.

26. Manning BD, Cantley LC. AKT/PKB signaling: navigating downstream. Cell. 2007;129(7):1261-74

27. Kohn AD, Takeuchi F, Roth RA. Akt, a pleckstrin homology domain containing kinase, is activated primarily by phosphorylation. J Biol Chem. 1996;271(36):21920-6.

28. Sarbassov DD, Guertin DA, Ali SM, Sabatini DM. Phosphorylation and regulation of Akt/PKB by the rictor-mTOR complex. Science. 2005;307(5712): 1098-101.

29. Mukhopadhyay D, Riezman H. Proteasome-independent functions of ubiquitin in endocytosis and signaling. Science. 2007;315(5809):201-5.

30. Pickart CM. Mechanisms underlying ubiquitination. Annu Rev Biochem. 2001;70:503-33.

31. Yang WL, Wang J, Chan CH, Lee SW, Campos AD, Lamothe B, Hur L, Grabiner BC, Lin X, Darnay BG, et al. The E3 ligase TRAF6 regulates Akt ubiquitination and activation. Science. 2009;325(5944):1134-8. 
32. Duan L, Perez RE, Lai X, Chen L, Maki CG. The histone demethylase JMJD2B is critical for p53-mediated autophagy and survival in Nutlin-treated cancer cells. J Biol Chem. 2019;294(23):9186-97.

33. Koppenol WH, Bounds PL, Dang CV. Otto Warburg's contributions to current concepts of cancer metabolism. Nat Rev Cancer. 2011;11(5):325-37.

34. Wan W, Peng K, Li M, Qin L, Tong Z, Yan J, Shen B, Yu C. Histone demethylase JMJD1A promotes urinary bladder cancer progression by enhancing glycolysis through coactivation of hypoxia inducible factor 1alpha. Oncogene. 2017;36(27):3868-77.

35. Backe MB, Jin C, Andreone L, Sankar A, Agger K, Helin K, Madsen AN, Poulsen SS, Bysani M, Bacos K, et al. The Lysine Demethylase KDM5B Regulates Islet Function and Glucose Homeostasis. J Diabetes Res. 2019; 2019:5451038.

36. Kim TD, Fuchs JR, Schwartz E, Abdelhamid D, Etter J, Berry WL, Li C, Ihnat MA, Li PK, Janknecht R. Pro-growth role of the JMJD2C histone demethylase in HCT-116 colon cancer cells and identification of curcuminoids as JMJD2 inhibitors. Am J Transl Res. 2014;6(3):236-47.

37. Fu LN, Wang YQ, Tan J, Xu J, Gao QY, Chen YX, Fang JY. Role of JMJD2B in colon cancer cell survival under glucose-deprived conditions and the underlying mechanisms. Oncogene. 2018;37(3):389-402.

38. Whiteman EL, Cho H, Birnbaum MJ. Role of Akt/protein kinase B in metabolism. Trends Endocrinol Metab. 2002;13(10):444-51.

39. Toker A, Yoeli-Lerner M. Akt signaling and cancer: surviving but not moving on. Cancer Res. 2006;66(8):3963-6.

40. Vanhaesebroeck B, Stephens L, Hawkins P. PI3K signalling: the path to discovery and understanding. Nat Rev Mol Cell Biol. 2012;13(3):195-203.

41. Han F, Li CF, Cai Z, Zhang X, Jin G, Zhang WN, Xu C, Wang CY, Morrow J, Zhang $S$, et al. The critical role of AMPK in driving Akt activation under stress, tumorigenesis and drug resistance. Nat Commun. 2018;9(1):4728.

42. Wang G, Long J, Gao Y, Zhang W, Han F, Xu C, Sun L, Yang SC, Lan J, Hou $Z$, et al. SETDB1-mediated methylation of Akt promotes its K63-linked ubiquitination and activation leading to tumorigenesis. Nat Cell Biol. 2019; 21(2):214-25.

43. Dasgupta M, Dermawan JK, Willard B, Stark GR. STAT3-driven transcription depends upon the dimethylation of K49 by EZH2. Proc Natl Acad Sci U S A. 2015;112(13):3985-90.

\section{Publisher's Note}

Springer Nature remains neutral with regard to jurisdictional claims in published maps and institutional affiliations.

Ready to submit your research? Choose BMC and benefit from:

- fast, convenient online submission

- thorough peer review by experienced researchers in your field

- rapid publication on acceptance

- support for research data, including large and complex data types

- gold Open Access which fosters wider collaboration and increased citations

- maximum visibility for your research: over $100 \mathrm{M}$ website views per year

At $\mathrm{BMC}$, research is always in progress.

Learn more biomedcentral.com/submissions 